\title{
Myopathy With SQSTM1 and TIA1 Variants: Clinical and Pathological Features
}

OPEN ACCESS

Edited by:

Edoardo Malfatti,

INSERM UMRS974 Institut de

Myologie, France

Reviewed by:

Enrico Bugiardini,

University College London,

United Kingdom

Rosanna Cardani,

Policlinico San Donato

(IRCCS), Italy

${ }^{*}$ Correspondence:

Gerald Pfeffer

gerald.pfeffer2@ucalgary.ca;

Margherita Milone

milone.margherita@mayo.edu

tThese authors have contributed equally to this work.

¥These authors have contributed equally as corresponding authors to this work.

Specialty section:

This article was submitted to Neuromuscular Diseases, a section of the journal

Frontiers in Neurology

Received: 06 December 2017

Accepted: 27 February 2018

Published: 19 March 2018

Citation:

Niu Z, Pontifex CS, Berini S, Hamilton LE, Naddaf E, Wieben $E$,

Aleff RA, Martens $K$, Gruber A,

Engel $A G$, Pfeffer $G$ and Milone $M$

(2018) Myopathy With SQSTM1 and

TIA1 Variants: Clinical and

Pathological Features.

Front. Neurol. 9:147.

doi: 10.3389/fneur.2018.00147

\begin{abstract}
Zhiyv Niu, ${ }^{1,2}$, Carly Sabine Pontifex ${ }^{3,4 \dagger}$, Sarah Berini ${ }^{5}$, Leslie E. Hamilton 6 , Elie Naddaf, Eric Wieben ${ }^{7}$, Ross A. Aleff ${ }^{7}$, Kristina Martens ${ }^{3,4}$, Angela Gruber ${ }^{8}$, Andrew G. Engel' Gerald Pfeffer ${ }^{3,4 *}$ and Margherita Milone ${ }^{5 * \neq}$

Department of Laboratory Medicine and Pathology, Mayo Clinic, Rochester, MN, United States, ${ }^{2}$ Department of Clinical Genomics, Mayo Clinic, Rochester, MN, United States, ${ }^{3}$ Department of Clinical Neurosciences, University of Calgary, Calgary, AB, Canada, ${ }^{4}$ Hotchkiss Brain Institute, University of Calgary, Calgary, AB, Canada, ${ }^{5}$ Department of Neurology, Mayo Clinic, Rochester, MN, United States, ${ }^{6}$ Department of Pathology and Laboratory Medicine, University of Calgary, Calgary, AB, Canada, ' Department of Biochemistry and Molecular Biology, Mayo Clinic, Rochester, MN, United States, ${ }^{8}$ Prevention Genetics, Marshfield, WI, United States
\end{abstract}

Objective: The aim of this study is to identify the molecular defect of three unrelated individuals with late-onset predominant distal myopathy; to describe the spectrum of phenotype resulting from the contributing role of two variants in genes located on two different chromosomes; and to highlight the underappreciated complex forms of genetic myopathies.

Patients and methods: Clinical and laboratory data of three unrelated probands with predominantly distal weakness manifesting in the sixth-seventh decade of life, and available affected and unaffected family members were reviewed. Next-generation sequencing panel, whole exome sequencing, and targeted analyses of family members were performed to elucidate the genetic etiology of the myopathy.

Results: Genetic analyses detected two contributing variants located on different chromosomes in three unrelated probands: a heterozygous pathogenic mutation in SQSTM1 (c.1175C>T, p.Pro392Leu) and a heterozygous variant in TIA1 (c.1070A>G, p.Asn357Ser). The affected fraternal twin of one proband also carries both variants, while the unaffected family members harbor one or none. Two unrelated probands (family 1 , II.3, and family $3, \| .1$ ) have a distal myopathy with rimmed vacuoles that manifested with index extensor weakness; the other proband (family 2, I.1) has myofibrillar myopathy manifesting with hypercapnic respiratory insufficiency and distal weakness.

Conclusion: The findings indicate that all the affected individuals have a myopathy associated with both variants in SQSTM1 and TIA1, respectively, suggesting that the two variants determine the phenotype and likely functionally interact. We speculate that the TIA1 variant is a modifier of the SQSTM1 mutation. We identify the combination of SQSTM1 and TIA1 variants as a novel genetic defect associated with myofibrillar myopathy and suggest to consider sequencing both genes in the molecular investigation of myopathy with rimmed vacuoles and myofibrillar myopathy although additional studies are needed to investigate the digenic nature of the disease.

Keywords: distal myopathy, myofibrillar myopathy, respiratory insufficiency, rimmed vacuoles, SQSTM1, TIA1 


\section{INTRODUCTION}

T-cell intracellular antigen-I (TIA1, chr.2) is a broadly expressed RNA-binding protein required for the formation of cytoplasmic stress granules, which play a crucial role in preventing misfolded protein accumulation (1). To date, a single TIA1 mutation (p.Glu384Lys) is known to cause a late-onset myopathy with rimmed vacuoles (MRVs) $(2,3)$. TIA1 mutations were recently detected in amyotrophic lateral sclerosis (ALS)/frontotemporal dementia (FTD) (1), but a subsequent study suggested exerting caution on the causality of the TIA1 variants in ALS (4).

Sequestosome-1 (SQSTM1, chr.5) is a scaffolding protein involved in multiple cellular processes, including apoptosis, cell survival, and autophagy. Its numerous domains allow SQSTM1 to serve as a frame for multiprotein complexes and regulator of ubiquitinated protein turnover (5). SQSTM1 mutations have been linked with a spectrum of phenotypes, including Paget disease of bone (PDB), ALS, FTD, and MRV (5-7). Hence, SQSTM1 mutations can lead to a multisystem proteinopathy although with incomplete penetrance. A single SQSTM1 mutation (c.1165+1G $>$ A) has been linked to MRV in one family and an unrelated patient (6). This patient was subsequently found to carry a coexisting TIA1 variant (c.1070A $>$ G, p.Asn357Ser) by Evila et al. (8). Evila et al's study reported also an additional sporadic MRV case carrying the same TIA1 variant but a different SQSTM1 mutation (p.Pro392Leu), which is known to cause PDB, ALS, and FTD, but the patient's phenotype was not illustrated (8). The authors raised the possibility of a digenic myopathy (8), which up to date has not been proven.

Herein, we describe the clinical and pathological phenotype of three unrelated probands harboring the combined heterozygous TIA1 and SQSTM1 variants in the setting of MRV or myofibrillar pathology, providing evidence that co-occurrence of these variants are associated with late-onset myopathy.

\section{PATIENTS AND METHODS}

\section{Patients}

Three patients with distal myopathy of unknown molecular defect were identified in the neuromuscular clinics during their clinical evaluation. Clinical history and findings and serological, electrophysiological, muscle pathological, and radiological data were reviewed. All patients provided written informed consent. The study was approved by the respective research ethics boards of Mayo Clinic (Institutional Review Board), Rochester, MN, USA and University of Calgary, Canada. We also obtained written and informed consent from the patients who gave specific permission to publish the data.

\section{Methods}

\section{Morphological Studies}

Conventional histochemical studies were performed on freshfrozen muscle biopsy $10-\mu \mathrm{m}$ thick sections and stained for hematoxylin-eosin, modified Gomori trichrome, NADH dehydrogenase, succinate dehydrogenase, cytochrome c oxidase, adenosine triphosphatase (at $\mathrm{pH} 4.3,4.6$, and 9.4), acid phosphatase, periodic acid-Schiff, oil red $\mathrm{O}$, non-specific esterase, and Congo red. Amyloid deposits were identified in Congo red stain sections viewed under rhodamine optics. For immunocytochemical studies cryostat sections $6-10 \mu \mathrm{m}$ thick were reacted with monoclonal antibodies against myotilin (Novocastra, Bannockburn, IL, USA), alpha-B-crystallin (Stressgen, Ann Arbor, MI, USA), desmin (Dako, Carpinteria, CA, USA), and dystrophin (Novocastra), as previously described (9). Families 1 and 2's proband's muscle sections were also reacted with monoclonal antibodies against TIA1 (Abcam, Cambridge, UK) and family 3's proband's muscle sections against TAR DNA binding protein 43 (Proteintech, Rosemont, IL, USA). The immunoreactive sites were visualized with secondary antibodies using immunoperoxidase (9).

\section{Clinical Molecular Genetic Studies}

Nineteen genes known to be causative of distal myopathy (ANO5, BAG3, CAV3, CRYAB, DES, DNAJB6, DYSF, FHL1, FLNC, GNE, LDB3, MATR3, MYH7, MYOT, SQSTM1, TCAP, TIA1, TTN and $V C P$ ) were tested by next-generation sequencing (NGS) in a commercial diagnostic laboratory (PreventionGenetics, Marshfield, WI, USA) in all three probands. All coding regions plus $20 \mathrm{bp}$ flanking non-coding regions of these genes were analyzed by a combined targeted NGS and Sanger sequencing, as previously described (10). In addition, SQSTM1 aCGH microarray analysis was performed in patient 1. ACTA1, SEPN1, and LMNA were analyzed by targeted NGS in family 2's proband because of the myofibrillar pathology.

\section{Expanded NGS Panel and Whole Exome Sequencing (WES) Studies}

As clinical gene analysis was thought to be non-diagnostic, additional molecular testing was performed at Mayo Clinic on genomic DNA samples. This included expanded NGS panels with a 141 gene myopathy panel and a 217 gene distal weakness panel (Data S1 in Supplementary Material) in family 2's proband and WES in family 1's proband. The expanded NGS panels test coding regions, splicing sites, and key regulatory regions of genes of interest using a custom reagent were developed by Mayo Clinic Genomics Laboratory and Agilent Technologies. Copy number variation $(\mathrm{CNV})$ analysis was conducted based on the depth of coverage using the PatternCNV pipeline in comparison with internal reference controls (11) (and manuscript in preparation). Variant interpretation was conducted according to ACMG guidelines. Gene list and variants detected are included in the supplemental data (Table S1 in Supplementary Material). WES was performed using Agilent SureSelect Human All exon V5 capture reagent, and it was sequenced on Illumina HiSeq at the Medical Genome Facility at Mayo Clinic. Routinely, $>95 \%$ of target region was sequenced at $>20 \times$. Sequencing results were processed using in house developed pipeline and annotated using Ingenuity (Qiagen Inc.) and Alamut Batch (Alamut) software for clinical interpretation $(12,13)$. DNA CNV analysis by PatternCNV and manual data review were conducted to scan for large copy number alteration (11). To identify variants of interest, we adopted an exome interpretation strategy described previously $(12,14)$. Briefly, after applying standard quality filter and tiered population frequency filters, variants in genes known to cause muscle diseases (based on OMIM, HGMD) were individually reviewed according to ACMG guidelines. Next, predicted high-impact variants and variants in 
muscle expressed genes (371 genes, accessed in December 2015, www.proteinatlas.org) (15) were examined as candidate findings. Detailed WES procedure and variant statistics are provided in supplemental data (Data S1 in Supplementary Material) due to the amount of variants detected.

\section{Testing of Family Members}

Targeted Sanger sequencing analyses were performed on probands and relative's available samples at Medical Genome Facility at Mayo Clinic for families 1 and 2 and at Dr. Pfeffer's laboratory at Hotchkiss Brain Institute for family 3.

Reference sequences were used: for TIA1, we used NM_022173.2, and for SQSTM1, we used NM_003900.4.

\section{RESULTS}

Pedigrees are represented in Figures 1A-C; clinical and laboratory findings are summarized in the Table $\mathbf{1}$ and illustrated in Figures 2 and 3.

\section{Family 1}

The proband is a 71-year-old man (Figure 1A) who noted difficulty extending his index finger, followed by more diffuse asymmetric distal weakness and atrophy in the upper limbs and, a year later, in the lower limbs. Recently, he has shown short-term memory deficits, difficulty findings words and organizing thoughts, and irritability. His neurological examination showed a predominant distal weakness mainly affecting finger extensors (Figures 2A,B) ankle dorsiflexors and toe extensors (Table 1). Toe flexors were spared. Creatine kinase (CK) value was mildly elevated (Table 1). Alkaline phosphatase was normal twice in the past 4 years, including bone isoenzyme. Needle electromyography (EMG) studies showed myopathic changes especially in the distal muscles with few fibrillation potentials and some neurogenic motor unit potentials in few muscles. Nerve conduction studies were normal. Shoulder MRI, performed to investigate joint pain, incidentally revealed focal fatty atrophy of the trapezius (Figure 2C). Deltoid biopsy showed an MRV (Figure 3A). Scattered vacuoles overreacted for TIA1. LAMP2 immunoreactivity was normal (data not shown). Neuropsychological assessment showed mildly impaired verbal learning/retention and semantic fluency, while brain MRI revealed mild generalized atrophy and leukoaraiosis (Figure 2D). There was no evidence of cardiac involvement by EKG and echocardiogram. Radiographs of axial and appendicular skeleton showed no features of PDB. His father, of Swiss descent, had PDB and manifested progressive distal weakness in his 70s. He was unable to extend his fingers and reportedly used his thumbs to hold a cup. He developed foot drop and lost ambulation in his early 80s. Proband's sister and children are asymptomatic, while a brother, who died of cancer, had hand weakness in the late $50 \mathrm{~s}$ (Figure 1A).

\section{Family 2}

The proband is an 80 - to 85 -year-old woman with progressive exertional dyspnea, followed by dysphagia, progressive distal limb weakness mainly involving ankle dorsiflexors, and myalgia. She had a pacemaker placed at the age of 65 years for bradycardia and atrial fibrillation and history of gastric bypass. She had no family history of weakness, PDB, or dementia. Her examination revealed predominantly distal asymmetric weakness (Figure 2E and Table 1). CK value was slightly elevated in one occasion $(231 \mathrm{U} / \mathrm{L}$; normal $<222 \mathrm{U} / \mathrm{L})$ and normal in another occasion. Alkaline phosphatase was normal twice, including bone isoenzyme. EMG study showed myopathic changes especially in the

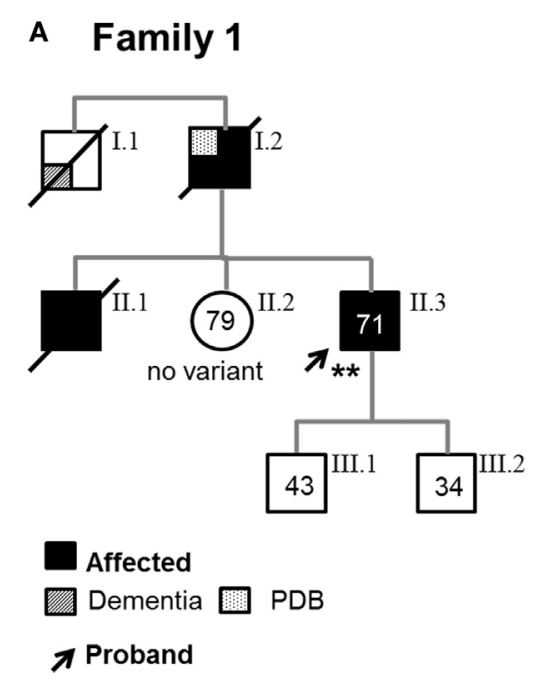

B Family 2

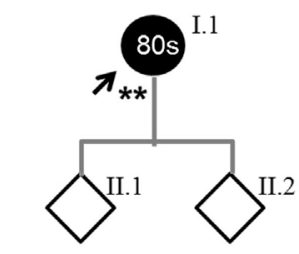

**Myopathy

TIA1 p. Asn357Ser (heterozygous)

SQSTM1 p.Pro392Leu (heterozygous)

$\diamond$ Gender and age undisclosed

\section{Family 3}

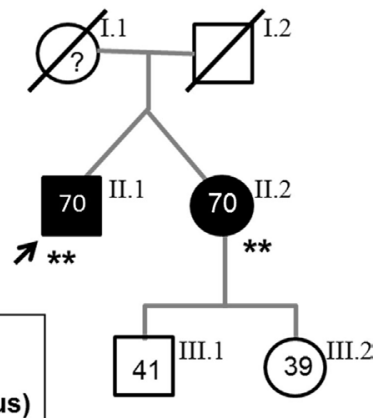

FIGURE 1 | Pedigrees. Arrows indicate probands, who are heterozygous for both the SQSTM1 and TIA1 variants and have a muscle biopsy-proven myopathy. (A) Family 1. Proband 1's asymptomatic sister (II.2) carries neither variant; the two asymptomatic sons carry neither variant nor the SQSTM1 variant. (B). Family 2. One of Proband 2's asymptomatic children harbors the TIA1 variant. (C). Family 3. Affected dizygotic twin siblings carry both TIA1 and SQSTM1 variants (only the probands underwent muscle biopsy). The asymptomatic son and daughter carry neither variant nor only the TIA1 variant. As some of the probands' children elected not to learn about their genetic status, carriers of specific variants are not indicated. (All living affected subjects were examined; the asymptomatic individuals were examined and had no weakness or were interviewed over the phone and denied weakness and symptoms suggestive of myopathy.) 
TABLE 1 | Clinical and laboratory findings of subjects carrying both the TIA1 and SQSTM1 variants.

\begin{tabular}{|c|c|c|c|c|}
\hline & Proband 1 & Proband 2 & Proband 3 & Patient 3.2 \\
\hline Age, sex & $71, \mathrm{M}$ & $80-85, F$ & $70, \mathrm{M}$ & $70, F$ \\
\hline Age at onset (years) & 65 & $70-75$ & 55 & 45 \\
\hline First symptom & Index extensor weakness & Dyspnea & Finger extensors weakness & $\begin{array}{l}\text { Finger extensor } \\
\text { weakness }\end{array}$ \\
\hline \multirow[t]{2}{*}{ Weakness, UL ${ }^{a}$} & $\begin{array}{l}\text { Finger extensors }>>\text { wrist } \\
\text { extensors }\end{array}$ & Finger extensors $>>$ wrist extensors & $\begin{array}{l}\text { Finger extensors }>>\text { wrist } \\
\text { extensors }\end{array}$ & $\begin{array}{l}\text { Finger extensors > } \\
>\text { wrist extensors }\end{array}$ \\
\hline & Shoulder girdle muscles (mild) & Shoulder girdle muscles (mild) & Arm muscles (mild) & Arm muscles (mild) \\
\hline \multirow[t]{2}{*}{ Weakness, LL ${ }^{a}$} & Toe extensors > ankle dorsiflexors & Toe extensors > ankle dorsiflexors & Toe extensors > ankle dorsiflexors & Left foot drop \\
\hline & Pelvic girdle (mild) & Pelvic girdle (mild) & Pelvic girdle (mild) & Pelvic girdle (mild) \\
\hline Gait & High stepping & High stepping & High stepping & High stepping \\
\hline Ability to walk on toes & No (spared toe flexors) & Mildly impaired & Mildly impaired & Mildly impaired \\
\hline Tendon reflexes & Absent at ankles & Absent at ankles & Normal & NA \\
\hline CK & 352-784 U/L (nl < 336) & 220-331 U/L (nl < 222) & Normal & NA \\
\hline Cardiac involvement ${ }^{\mathrm{b}}$ & None & Bradycardia, atrial fibrillation, pacemaker & None & NA \\
\hline $\begin{array}{l}\text { Respiratory } \\
\text { involvement }\end{array}$ & None & $\begin{array}{l}\downarrow \text { Max exp press, nocturnal } \mathrm{O}_{2} \\
\text { desaturation }\end{array}$ & None & NA \\
\hline EMG findings & $\begin{array}{l}\text { Myopathic; mild neurogenic } \\
\text { changes distally }\end{array}$ & Myopathic & Myopathic & NA \\
\hline Muscle imaging & Atrophy trapezius (MRI)c & $\begin{array}{l}\text { Atrophy anterior compartment; fatty } \\
\text { infiltration lower calf muscles (CT) }\end{array}$ & $\begin{array}{l}\text { Fatty infiltration adductor } \\
\text { magnus, vastus medialis, anterior } \\
\text { compartment, and medial } \\
\text { gastrocnemius (MRI) }\end{array}$ & NA \\
\hline $\mathrm{PBD}^{d}$ & None & None & NA & NA \\
\hline MRI brain & Mild generalized atrophy & NA & Normal & NA \\
\hline $\begin{array}{l}\text { Cognitive } \\
\text { assessment }\end{array}$ & $\begin{array}{l}\text { Mild impaired verbal learning/ } \\
\text { retention, semantic fluency }\end{array}$ & Normal & Normal & Normal \\
\hline
\end{tabular}

weakest muscles and diaphragm with fibrillation potentials in the tibialis anterior. Nerve conduction studies and $2 \mathrm{~Hz}$ repetitive nerve stimulations of the ulnar and spinal accessory nerves were normal. CT of the leg showed distal fatty atrophy of the muscles (Figure 2F). Pulmonary function tests were significant for reduced maximal expiratory pressure to $63 \%$ of predicted, while overnight oximetry showed desaturation to $80 \%$ (patient declined nocturnal polysomnography). Tibialis anterior biopsy (Figures 3B-F) showed features of myofibrillar myopathy (16). In ATPase reacted sections, there was grouping of type 1 fiber in several fascicles suggesting reinnervation (data not shown). Proband 2 was patient \# 9 in a case series of myopathy with respiratory insufficiency (17). No bone abnormalities were evident by leg CT or hip radiograph. EKG revealed a controlled ventricular rate in the setting of electronic atrial pacemaker; 24-h-Holter monitoring and echocardiogram were unrevealing.

\section{Family 3, Proband (Patient II.1)}

The proband is a 70-year-old man of British descent (Figure 1C) who developed difficulty fully extending his index finger bilaterally at the age of 55 years. A few years later, he noted that he would catch his toes on the curb while walking and had a high-stepping gait. He was found to have mild finger extensor and ankle dorsiflexor weakness. A muscle biopsy showed MRV (Figures 3G-I). His weakness did not significantly progress; he remained ambulant. At the age of 70 years, he has bilateral distal atrophy of intrinsic hand and foot muscles and mild left scapular winging (Table 1). The weakness affected preferentially distal extensor muscles. CK level was normal. EMG study showed myopathic changes. Muscle MRI showed fatty infiltration predominantly of adductor magnus and medial vastus lateralis in the thighs, anterior compartment muscles, and medial gastrocnemius in the legs (Figure 2G). Patient had no evidence of cardiac dysfunction by EKG and echocardiogram. His mother had difficulty with stairs around age 60 but did not seek medical attention and remained ambulant until death at age 89. His sister (Patient 3.2 below) has weakness. No other family members have any neurological symptoms.

\section{Family 3, Patient II.2}

She is a 70-year-old woman and fraternal twin of proband II.1 (Figure 1C). She developed weakness of right index finger extension in her mid 40's, followed by left foot drop in her mid 50's. 

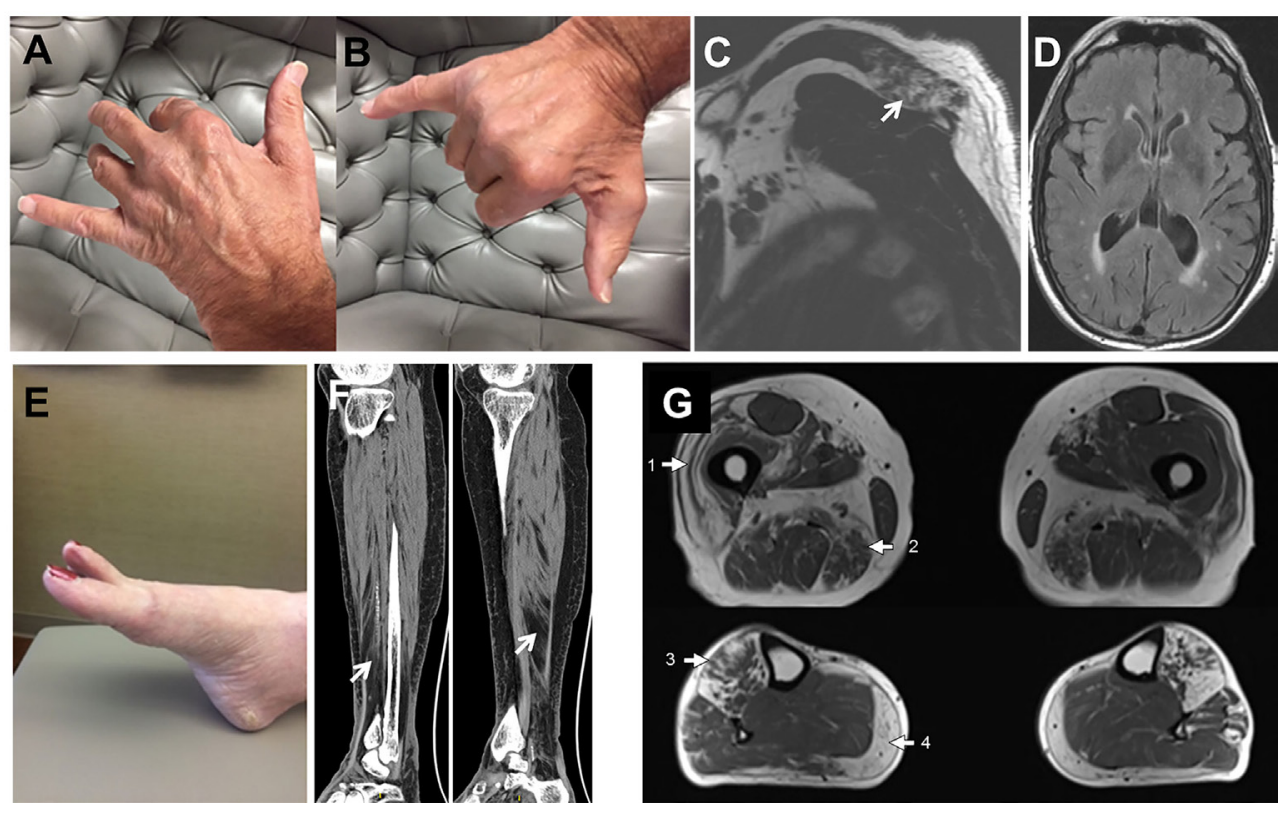

FIGURE 2 | Patient's photographs and radiological images. Proband 1 (A-D). Photographs illustrating the predominant weakness of digit II to IV extensors with spared I and $V$ digit extensors (A,B). MRI images demonstrating focal fatty atrophy (arrow) involving the left posterior trapezius muscle (C). T2 FLAIR brain MRI images showing mild generalized atrophy with patchy and confluent periventricular white matter hyperintensities suggestive of leukoaraiosis (D). Proband 2 (E,F). Photograph demonstrating the hanging big toe (E). CT images revealing moderate-marked fatty atrophy involving the anterior compartment musculature of the right lower leg overlying the distal third of the tibia and fatty infiltration of the lower calf muscle (F). Proband 3 (G). Muscle MRI showing fatty infiltration of muscles in a geographic distribution, indicated by numbered arrows. The predominantly affected muscles include the vastus lateralis (1) and adductor magnus (2) in the thighs and anterior compartment muscles (3) and medial gastrocnemius (4) in the lower legs.

At the age of 60 years, she noted slowly progressive left finger extensor weakness and cannot extend her fingers against gravity at 70 years of age. Recently, she has developed right finger extensor weakness (Table 1). She has two children and two grandchildren with no neurological symptoms.

\section{Molecular Data}

All three probands carry two heterozygous variants: SQSTM1, c.1175C > T (p.Pro392Leu), and TIA1, c.1070A > G (p.Asn357Ser). None of the unaffected family members harbor both variants (Figure 1). The TIA1 variant and SQSTM1 variants have been reported in multiple databases. The SQSTM1 variant is designated as rs104893941 in dbSNP and reported at allele frequencies of 0.0009 in the Exome Aggregation Consortium database (ExAC) (18), 0.0024 in 1,000 Genomes Project database (TGP) (19), and 0.0015 in the NHLBI GO Exome Sequencing Project (GO-ESP) (accessed January 23, 2018) $(19,20)$ and is more frequent in certain European populations. The TIA1 variant is designated as rs116621885 and reported at allele frequencies of 0.0071 in ExAC, 0.0016 in TGP, and 0.0068 in GO-ESP (accessed January 23, 2018). We examined the genotype data in the TGP to determine whether these variants coincide in controls (21). None of 2,504 self-declared healthy individuals in TGP has both TIA1, c.1070A > G (p.Asn357Ser) and SQSTM1, c.1175C > T (p.Pro392Leu). No other pathogenic or suspected pathogenic variants in genes associated with muscle diseases were identified in the proband of family 2 by expanded NGS panel studies or in the proband of family 1 by WES analysis.
We are aware of a prior study in which this SQSTM1 mutation may be part of a common founder haplotype including the following four loci: [Chr5: 179260153C/T, refSNP ID rs4935; Chr5: 179260213G/A, rs4797; Chr5: 179264731T/C, rs10277; Ch5: $179264915 \mathrm{G} / \mathrm{T}, \mathrm{rs} 1065154$ (22)]. On the basis of our available sequencing data, we attempted to infer the haplotype of the SQSTM1 mutation for each family. The proband from Family 1 is consistent with the $\mathrm{H} 1$ haplotype based on the presence of homozygous genotypes for rs4935 and rs4797 although this is not definitive because the rs 10277 and rs 1065154 polymorphisms were not covered. The haplotype of the proband from Family 2 could not be determined based on the available genotype data. For Family 3 , sequencing data were available for four family members, and we manually reconstructed the haplotype assuming the minimal number of recombinations. The result indicated that Family 3's haplotype was consistent with either the $\mathrm{H} 2$ or the $\mathrm{H} 5$ haplotype described in the study by Lucas et al. (22). On the basis of these results, our three families have at least two different haplotypes associated with the SQSTM1 mutation, indicating that this unique phenotype is not a haplotype-specific effect, as well as demonstrating that these families are not remotely related to each other.

\section{DISCUSSION}

We present the first detailed clinical and pathologic data from three unrelated families with predominant distal myopathy associated with a known pathologic variant in SQSTM1 (p.Pro392Leu) and a variant in TIA1 (p.Asn357Ser). At the time of this report, 


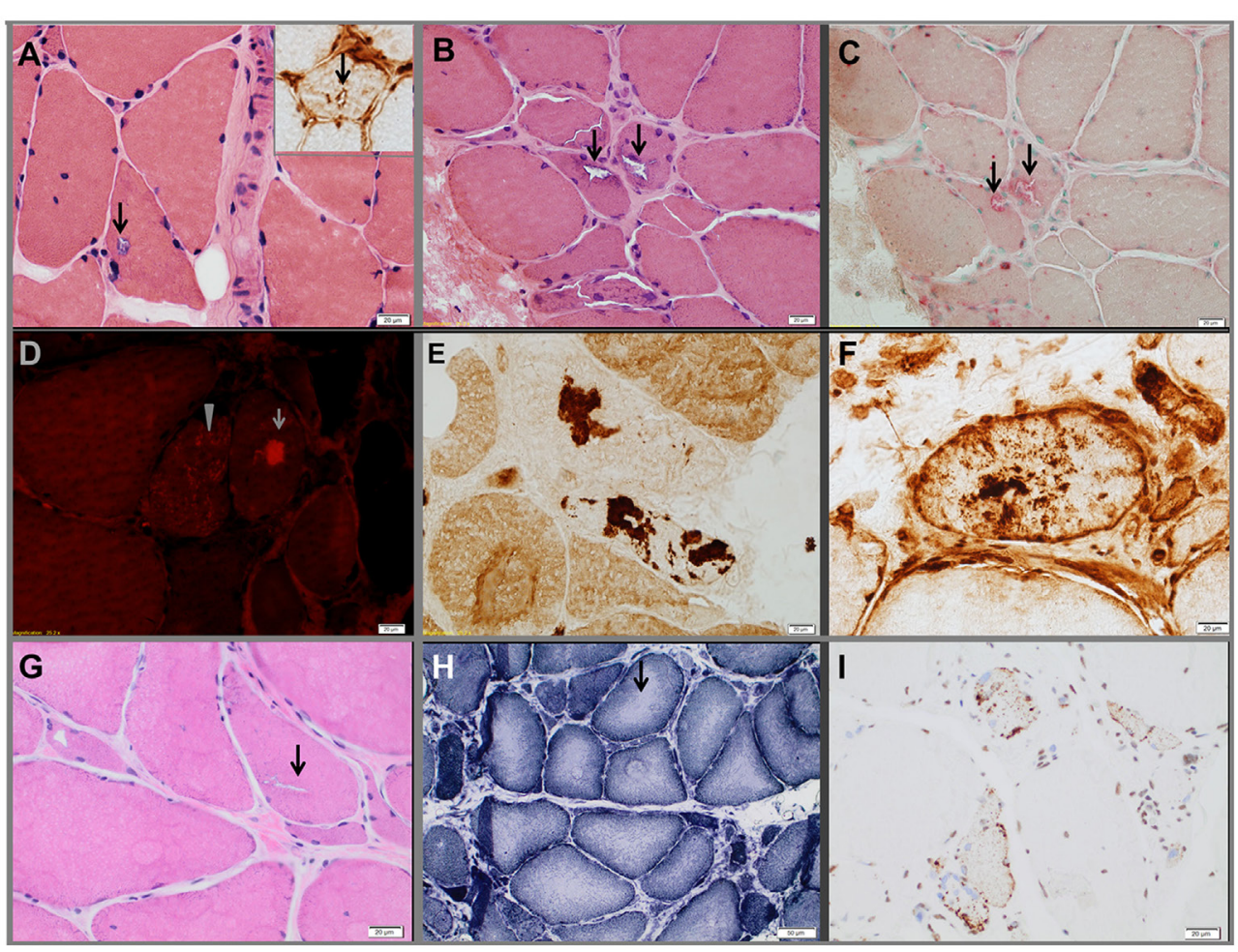

FIGURE 3 | Muscle biopsies from the three probands. Proband 1 (A). Hematoxylin-eosin-stained section demonstrating a vacuole (arrows) rimmed by membranous material. The insert in the upper right corner shows a TIA1-positive inclusion within a vacuole. Proband 2 (B-F). Hematoxylin-eosin-stained section (B) showing rimmed vacuoles (arrows), which strongly overreact for acid phosphatase (C). Extravacuolar large (arrow) or small congophilic inclusions (bright red dots within muscle fiber pointed by arrow head) (D) are present in structurally abnormal fibers (congo red stained section viewed under rhodamine optics). Several fibers demonstrated focal accumulation (dark stain) of myotilin [(E), representative fiber], TIA1 [(F), representative fiber], alpha-B crystallin, and desmin (data not shown). The pathological findings are suggestive of myofibrillar myopathy. Proband 3 (G-I), hematoxylin-eosin-stained section showing chronic myopathic changes and rimmed vacuoles (arrow) (G). NADH-TR-stained section revealing "rubbed-out" areas of decreased oxidative enzyme reactivity (arrow, representative fiber) in multiple myofibers (H). Several fibers demonstrating mislocalized cytoplasmic granules of TDP-43 staining (dark stain) (I).

only a single prior myopathy case with the same genetic variants has been reported, but the clinical and myopathological features were not illustrated (8). There are also two further cases of MRV having the same TIA1 variant but a different SQSTM1 mutation (c.1165+1G>A) (8), one of whom was previously reported as having a SQSTM1-MRV (6). Although the causality of the coexisting SQSTM1 and TIA1 variants in myopathy has not been proven, our affected individuals from three unrelated family provide further support to the digenic nature of this myopathy. In addition, we have identified a novel genetic defect (the combined SQSTM1 and TIA1 variants) associated with myofibrillar pathology, which to our knowledge has not been previously described in association with TIA1 and SQSTM1 variants, in isolation or in combination.

The contributing role of the two detected variants in causing the myopathy is supported by the following: (1) the SQSTM1 p.Pro392Leu mutation is a known pathogenic variant previously associated with ALS, FTD, and/or PDB, and although by itself it has never been reported in association with a myopathy, a different SQSTM1 mutation was detected in an MRV case (6); (2) the TIA1 variant p.Asn357Ser (designated as rs 116621885 in dbSNP) is less likely to be causative by itself of a dominant disease, given the population frequency of $0.71 \%$ in ExAC databases and has never been shown to segregate with disease, except in combination with SQSTM1 mutations $(8,18)$; (3) all our four patients from three independent families with myopathy have both genetic variants, and the likelihood that these two variants occurring in three families by chance (given they are on different chromosomes) is extremely small; (4) co-segregation of these two variants is not detected in the self-declared healthy subjects of the TGP database (21); (5) exome sequencing in proband 1 and a 362-gene neuromuscular disorders panel in proband 2 did not identify an alternative genetic etiology; and (6) there is plausible biological rationale for an interaction between variants in TIA 1 and SQSTM1 and their interplay in causing myopathy because both genes are involved in protein quality control via stress granule assembly and autophagy, respectively. SQSTM1 is localized adjacent to stress granules for extraction of ubiquitinated proteins for autophagy (6), suggesting the possibility that genetic variations in both TIA1 and SQSTM1 can interact and disrupt this pathway. However, molecular and functional studies to dissect the likely interaction between TIA1 and SQSTM1 would shed light on the pathomechanism of the myopathy. Given that the TIA 1 p.Asn357Ser variant is relatively common, and therefore likely not pathogenic by itself, we speculate that the TIA1 variant may have a modifier effect on the phenotype related to 
the pathogenic SQSTM1 mutations. The digenic nature of the myopathy is now supported by the presence of myopathy in five unrelated probands [three from this report and two from the study by Evila et al. (8)], all carrying a pathogenic SQSTM1 mutation and the TIA1 p.Asn357Ser variant.

The patients reported here show phenotypic variability, a wellrecognized feature of late-onset hereditary myopathies, although three of them manifested with index extensor weakness, a classic feature of TIA1 myopathy. The myopathic respiratory insufficiency and atrial fibrillation (although the latter finding could an incidental finding) in family 2's proband emphasize the need to investigate respiratory and cardiac function in future cases of SQSTM1/TIA1 myopathy.

Myofibrillar myopathy, detected in family 2's proband, has not been previously described in association with TIA1 or SQSTM1 variants, in isolation or in combination. Such finding is not surprising because of the known pathological overlap between MRV and myofibrillar myopathy. However, in light of our observation, we suggest that SQSTM1/TIA analysis is included in the molecular investigations of myofibrillar myopathies.

The cognitive impairment in family 1 may reflect the previously reported deleterious effects of the TIA1 and SQSTM1 variants on brain, as mutations in both genes have been identified in ALS and FTD (1). In addition, one could speculate that the histopathological features of reinnervation observed in proband 2's muscle biopsy might signal a coexisting neurogenic component due to the underlying genetic makeup.

Large genomic sequencing projects have enabled studies on the causes of complex genetic disease and often broad clinical manifestations $(18,21)$. Recent studies have highlighted that digenic interactions in genetic diseases, especially in neuromuscular disorders, may be more frequent than previously appreciated (23-25). There is indeed emerging evidence that many genetic factors can contribute to the phenotype variability, especially in complex neuromuscular disease (25-27). The cases described here support the importance of considering the interaction among multiple genetic variants in determining the patient's phenotype, disease mechanisms, and modality of inheritance. The lack of functional studies is certainly a limiting feature of the study, and further investigations will be needed to prove the digenic nature of the myopathy and the

\section{REFERENCES}

1. Mackenzie IR, Nicholson AM, Sarkar M, Messing J, Purice MD, Pottier C, et al. TIA1 mutations in amyotrophic lateral sclerosis and frontotemporal dementia promote phase separation and alter stress granule dynamics. Neuron (2017) 95(4):808.e-16.e. doi:10.1016/j.neuron.2017.07.025

2. Hackman P, Sarparanta J, Lehtinen S, Vihola A, Evila A, Jonson PH, et al. Welander distal myopathy is caused by a mutation in the RNA-binding protein TIA1. Ann Neurol (2013) 73(4):500-9. doi:10.1002/ana.23831

3. Klar J, Sobol M, Melberg A, Mabert K, Ameur A, Johansson AC, et al. Welander distal myopathy caused by an ancient founder mutation in TIA1 associated with perturbed splicing. Hum Mutat (2013) 34(4):572-7. doi:10.1002/ humu. 22282

4. Van Der Spek RA, Van Rheenen W, Pulit SL, Kenna KP, Ticozzi N, Kooyman M, et al. Reconsidering the causality of TIA1 mutations in ALS. Amyotroph Lateral Scler Frontotemporal Degener (2018) 19(1-2):1-3. doi:10.1080/2167 8421.2017 .1413118 deleterious effects of the combined SQSTM1 and TIA1 variants in myopathy.

\section{ETHICS STATEMENT}

All patients provided written informed consent. The study was approved by the respective research ethics boards of Mayo Clinic (Institutional Review Board), Rochester MN, USA and University of Calgary, AB, Canada. We also obtained written and informed consent from the patients who gave specific permission to publish the data.

\section{AUTHOR CONTRIBUTIONS}

Study concept and design: MM, ZN, and GP. Acquisition, analysis, or interpretation of data: all authors. Drafting of the manuscript: ZN, GP, and MM. Critical revision of the manuscript for important intellectual content: $\mathrm{ZN}, \mathrm{AE}, \mathrm{LH}, \mathrm{EN}, \mathrm{GP}, \mathrm{MM}$. Obtained funding: MM. ZN and CP contributed equally. GP and MM contributed equally.

\section{ACKNOWLEDGMENTS}

The authors are extremely grateful to the patients for their support, thrust and willingness to share their data with the scientific community. The authors thank Bruce Eckloff and Yanhong Wu, PhD (Medical Genome Facility) for technical support; the Biomedical Statistics and Informatics team for preliminary data analysis; and Mrs. Carey Huebert (research coordinator) at Mayo Clinic.

\section{FUNDING}

This work was funded through a generous gift from a Mayo Clinic benefactor and the Mayo Clinic Center for Individualized Medicine (RFA 48.07).

\section{SUPPLEMENTARY MATERIAL}

The Supplementary Material for this article can be found online at https://www.frontiersin.org/articles/10.3389/fneur.2018.00147/ full\#supplementary-material.

5. Hocking LJ, Lucas GJ, Daroszewska A, Mangion J, Olavesen M, Cundy T, et al. Domain-specific mutations in sequestosome 1 (SQSTM1) cause familial and sporadic Paget's disease. Hum Mol Genet (2002) 11(22):2735-9. doi:10.1093/ hmg/11.22.2735

6. Bucelli RC, Arhzaouy K, Pestronk A, Pittman SK, Rojas L, Sue CM, et al. SQSTM1 splice site mutation in distal myopathy with rimmed vacuoles. Neurology (2015) 85(8):665-74. doi:10.1212/WNL.0000000000001864

7. Rubino E, Rainero I, Chio A, Rogaeva E, Galimberti D, Fenoglio P, et al. SQSTM1 mutations in frontotemporal lobar degeneration and amyotrophic lateral sclerosis. Neurology (2012) 79(15):1556-62. doi:10.1212/ WNL.0b013e31826e25df

8. Evila A, Arumilli M, Udd B, Hackman P. Targeted next-generation sequencing assay for detection of mutations in primary myopathies. Neuromuscul Disord (2016) 26(1):7-15. doi:10.1016/j.nmd.2015.10.003

9. Selcen D, Ohno K, Engel AG. Myofibrillar myopathy: clinical, morphological and genetic studies in 63 patients. Brain (2004) 127(Pt 2):439-51. doi:10.1093/ brain/awh052 
10. Brand P, Dyck PJ, Liu J, Berini S, Selcen D, Milone M. Distal myopathy with coexisting heterozygous TIA1 and MYH7 Variants. Neuromuscul Disord (2016) 26(8):511-5. doi:10.1016/j.nmd.2016.05.012

11. Wang C, Evans JM, Bhagwate AV, Prodduturi N, Sarangi V, Middha M, et al. PatternCNV: a versatile tool for detecting copy number changes from exome sequencing data. Bioinformatics (2014) 30(18):2678-80. doi:10.1093/ bioinformatics/btu363

12. Martinez-Thompson JM, Niu Z, Tracy JA, Moore SA, Swenson A, Wieben ED, et al. Autosomal dominant calpainopathy due to heterozygous CAPN3 c.643_663del21. Muscle Nerve (2017). doi:10.1002/mus.25970

13. McKenna A, Hanna M, Banks E, Sivachenko A, Cibulskis K, Kernytsky A, et al. The Genome Analysis Toolkit: a MapReduce framework for analyzing next-generation DNA sequencing data. Genome Res (2010) 20(9):1297-303. doi:10.1101/gr.107524.110

14. Yang Y, Muzny DM, Reid JG, Bainbridge MN, Willis A, Ward PA, et al. Clinical whole-exome sequencing for the diagnosis of Mendelian disorders. $N$ Engl $J$ Med (2013) 369(16):1502-11. doi:10.1056/NEJMoa1306555

15. Lindskog C, Linne J, Fagerberg L, Hallstrom BM, Sundberg CJ, Lindholm M, et al. The human cardiac and skeletal muscle proteomes defined by transcriptomics and antibody-based profiling. BMC Genomics (2015) 16:475. doi:10.1186/s12864-015-1686-y

16. Selcen D. Myofibrillar myopathies. Neuromuscul Disord (2011) 21(3):161-71. doi:10.1016/j.nmd.2010.12.007

17. Naddaf E, Milone M. Hereditary myopathies with early respiratory insufficiency in adults. Muscle Nerve (2017) 56(5):881-6. doi:10.1002/mus.25602

18. Lek M, Karczewski KJ, Minikel EV, Samocha KE, Banks E, Fennell T, et al. Analysis of protein-coding genetic variation in 60,706 humans. Nature (2016) 536(7616):285-91. doi:10.1038/nature19057

19. The 1000 Genomes Project Consortium, Auton A, Brooks LD, Durbin RM, Garrison EP, Kang HM, et al. A global reference for human genetic variation. Nature (2015) 526(7571):68-74. doi:10.1038/nature15393

20. Auer PL, Reiner AP, Wang G, Kang HM, Abecasis GR, Altshuler D, et al. Guidelines for large-scale sequence-based complex trait association studies: lessons learned from the NHLBI exome sequencing project. Am J Hum Genet (2016) 99(4):791-801. doi:10.1016/j.ajhg.2016.08.012
21. The 1000 Genomes Project Consortium, Abecasis GR, Auton A, Brooks LD, DePristo MA, Durbin RM, et al. An integrated map of genetic variation from 1,092 human genomes. Nature (2012) 491(7422):56-65. doi:10.1038/nature11632

22. Lucas GJ, Hocking LJ, Daroszewska A, Cundy T, Nicholson GC, Walsh JP, et al. Ubiquitin-associated domain mutations of SQSTM1 in Paget's disease of bone: evidence for a founder effect in patients of British descent. J Bone Miner Res (2005) 20(2):227-31. doi:10.1359/JBMR.041106

23. Posey JE, Harel T, Liu P, Rosenfeld JA, James RA, Coban Akdemir ZH, et al. Resolution of disease phenotypes resulting from multilocus genomic variation. N Engl J Med (2017) 376(1):21-31. doi:10.1056/NEJMoa1516767

24. Posey JE, Rosenfeld JA, James RA, Bainbridge M, Niu Z, Wang X, et al. Molecular diagnostic experience of whole-exome sequencing in adult patients. Genet Med (2016) 18(7):678-85. doi:10.1038/gim.2015.142

25. Gonzaga-Jauregui C, Harel T, Gambin T, Kousi M, Griffin LB, Francescatto L, et al. Exome sequence analysis suggests that genetic burden contributes to phenotypic variability and complex neuropathy. Cell Rep (2015) 12(7):1169-83. doi:10.1016/j.celrep.2015.07.023

26. Gazzo AM, Daneels D, Cilia E, Bonduelle M, Abramowicz M, Van Dooren S, et al. DIDA: a curated and annotated digenic diseases database. Nucleic Acids Res (2016) 44(D1):D900-7. doi:10.1093/nar/gkv1068

27. Meola G, Cardani R. Myotonic dystrophy type 2 and modifier genes: an update on clinical and pathomolecular aspects. Neurol Sci (2017) 38(4):535-46. doi:10.1007/s10072-016-2805-5

Conflict of Interest Statement: Author AG was employed by company Prevention Genetics. All other authors declare no competing interests.

Copyright (C) 2018 Niu, Pontifex, Berini, Hamilton, Naddaf, Wieben, Aleff, Martens, Gruber, Engel, Pfeffer and Milone. This is an open-access article distributed under the terms of the Creative Commons Attribution License (CC BY). The use, distribution or reproduction in other forums is permitted, provided the original author(s) and the copyright owner are credited and that the original publication in this journal is cited, in accordance with accepted academic practice. No use, distribution or reproduction is permitted which does not comply with these terms. 